Jeunes adultes issus de l'immigration et marché du travail. Logiques d'orientation professionnelle Young Adults from Immigrant Backgrounds and the Labour Market. Professional Orientation and Their Underlying Rationales

\author{
Fahimeh Darchinian, Marie-Odile Magnan et Fasal Kanouté
}

Volume 17, 2017

La « diversité » ethnoculturelle au Québec : rapports identitaires, discrimination, exclusion et racisme

URI : https://id.erudit.org/iderudit/1047980ar

DOI : https://doi.org/10.7202/1047980ar

Aller au sommaire du numéro

\section{Éditeur(s)}

Groupe de recherche diversité urbaine CEETUM

\section{ISSN}

1913-0694 (imprimé)

1913-0708 (numérique)

Découvrir la revue

\section{Citer cet article}

Darchinian, F., Magnan, M.-O. \& Kanouté, F. (2017). Jeunes adultes issus de l'immigration et marché du travail. Logiques d'orientation professionnelle. Diversité urbaine, 17, 113-132. https://doi.org/10.7202/1047980ar
Résumé de l'article

Cet article présente les résultats d'une recherche exploratoire portant sur le travail de négociation de jeunes adultes montréalais issus de l'immigration en ce qui a trait à la construction de leurs logiques d'orientation professionnelle. Dans la littérature scientifique, le passage vers le marché du travail chez cette population a surtout été étudié en fonction du taux d'accès à l'emploi. Basé sur la méthode des récits de vie, le corpus qualitatif comprend 25 jeunes adultes issus de l'immigration qui comptent deux ans d'expérience sur le marché du travail montréalais. L'angle interprétatif de la recherche rend compte des relations sociales et des déterminations sociales négociées et représentées dans le discours des jeunes adultes interrogés. Les résultats montrent que les vécus de discrimination (racisme, linguicisme, intolérance religieuse) amènent plusieurs de ces jeunes adultes à s'intégrer dans les milieux anglophones afin de s'éloigner des milieux de travail francophones. 


\title{
Jeunes adultes issus de l'immigration et marché du travail. Logiques d'orientation professionnelle
}

\author{
Young Adults from Immigrant Backgrounds \\ and the Labour Market. \\ Professional Orientation and Their Underlying \\ Rationales
}

\author{
FAHIMEH DARCHINIAN \\ Département d'administration et fondements de l'éducation, Université de \\ Montréal, fahimeh.darchinian@umontreal.ca
}

\section{MARIE-ODILE MAGNAN \\ Département d'administration et fondements de l'éducation, Université de Montréal, marie-odile.magnan@umontreal.ca}

\section{FASAL KANOUTÉ}

Département de psychopédagogie et d'andragogie, Université de Montréal, fasal.kanoute@umontreal.ca

\begin{abstract}
RÉSUMÉ = Cet article présente les résultats d'une recherche exploratoire portant sur le travail de négociation de jeunes adultes montréalais issus de l'immigration en ce qui a trait à la construction de leurs logiques d'orientation professionnelle. Dans la littérature scientifique, le passage vers le marché du travail chez cette population a surtout été étudié en fonction du taux d'accès à l'emploi. Basé sur la méthode des récits de vie, le corpus qualitatif comprend 25 jeunes adultes issus de l'immigration qui comptent deux ans d'expérience sur le marché du travail montréalais. L'angle interprétatif de la recherche rend compte des relations sociales et des déterminations sociales négociées et représentées dans le discours des jeunes adultes interrogés. Les résultats montrent que les vécus de discrimination (racisme, linguicisme, intolérance religieuse) amènent plusieurs de ces jeunes adultes à s'intégrer dans les milieux anglophones afin de s'éloigner des milieux de travail francophones.
\end{abstract}

ABSTRACT $₫$ This article presents the results of exploratory research on how young adults from immigrant backgrounds in Montreal negotiate their professional career paths. In the scientific literature, their transition to the labor market has been problematized mainly with regard to employment access. Based on the life history method, our qualitative research focuses on 25 young adults from immigrant backgrounds who have two years of experience in the Montreal labor market. The interpretive angle of the research takes into account the social relations and determinations, negotiated and represented in the discourse of interviewed young adults. 
The results show that the experiences of discrimination (racism, linguicism, religious intolerance) drive many of these young adults to integrate themselves into Englishspeaking environments by distancing themselves from French-language environments.

MотS CLÉs = Orientation professionnelle, logiques d'orientation, discrimination, jeunes adultes issus de l'immigration, minorités visibles.

KEYWORDS = Professional orientation, orientation rationales, discrimination, young adults of immigrant background, visible minorities.

\section{Introduction}

En 2015, le Canada a accueilli 271845 nouveaux arrivants, soit plus qu'en 2014 (260 404) (Statistique Canada 2016). Pour sa part, le Québec a reçu 49024 nouveaux arrivants, dont $76 \%$ se sont installés sur l'île de Montréal (ministère de l'Immigration, de la Diversité et de l'Inclusion [MIDI] 2016). Pendant la même période, toujours à Montréal, 62,8 \% des élèves du primaire et du secondaire étaient issus de l'immigration, c'est-à-dire soit nés à l'étranger de parents nés à l'étranger (22,1\%), nés au Québec de parents nés à l'étranger $(29,7 \%)$, ou encore nés au Québec et ayant un parent né à l'étranger (11,0\%) (Comité de gestion de la taxe scolaire de l'île de Montréal [CGTSIM] 2016). Par ailleurs, à Montréal, la proportion d'élèves dont la langue maternelle n'est ni le français ni l'anglais était de 42,69\% (ibid.).

L'objectif d'égalité des chances défini par le Canada et le Québec canalise l'attention des chercheurs sur les thèmes de la réussite scolaire et de l'insertion professionnelle des jeunes issus de l'immigration (Mc Andrew 2010). Jusqu'à présent, leur passage vers le marché du travail a été principalement documenté par des données statistiques sur le taux d'accès à l'emploi. De manière générale, les résultats des études empiriques soulignent que les jeunes adultes canadiens et québécois issus de l'immigration réussissent à s'insérer sur le marché du travail (Guìrra 2016; Mc Andrew et al. 2011), et ce, même en considérant les différences entre les groupes et les sous-groupes (Kamanzi et Murdoch 2011; Mc Andrew 2010).

Ces jeunes, en tant qu'acteurs sociaux, sont aptes à construire leur expérience de vie et à donner un sens à leur parcours. Pourtant, à ce jour, peu d'études ont porté sur le travail de négociation des jeunes adultes issus de l'immigration lors de la construction de leurs expériences d'orientation professionnelle (Bélanger et al. 2011; Langlois 2009; Pagé et Lamarre 2010). Cette recherche s'inscrit dans le champ de la sociologie 
interprétative, où est pris en compte le rôle des individus dans la construction de leur expérience de vie, tout en reconnaissant celui, contraignant, des déterminations sociales (Lahire 2005). En définitive, une réalité collective alimente les expériences humaines, comme c'est le cas pour les expériences d'orientation professionnelle des jeunes adultes issus de l'immigration. Or, les individus n'agissent que dans le réseau des relations qu'ils établissent avec autrui (Becker 2008). L'acteur social, par son va-etvient dans le réseau complexe des déterminations sociales, négocie nécessairement ses relations sociales (Liu et Emirbayer 2016).

C'est à partir d'une analyse relationnelle de l'expérience d'orientation professionnelle que nous proposons de repérer des types de logiques d'orientation (Lahire 2013; Liu et Emirbrayer 2016), en tenant compte de la dynamique relationnelle entre les jeunes adultes issus de l'immigration et la population native du Québec (Juteau 2015; Lamont et Molnár 2002). Pour ce faire, nous analysons les récits de vie de 25 jeunes adultes montréalais issus de l'immigration. Notre analyse typologique met en exergue trois types de logiques d'orientation professionnelle qu'ils ont développés afin de construire leur expérience d'orientation professionnelle. Avant de plonger au cœur de l'analyse des données, il importe de présenter une synthèse des recherches menées sur l'expérience de la transition vers le marché du travail chez les jeunes adultes issus de l'immigration dans plusieurs contextes nationaux, et plus particulièrement au Québec et à Montréal. Ensuite, nous présenterons notre cadre d'analyse (Lahire 2013; Liu et Emirbayer 2016) et notre cadre méthodologique interprétatif. Nous terminerons par une interprétation des résultats à l'aide d'une analyse typologique des logiques d'orientation professionnelle. Nous montrerons qu'à l'étape de la transition vers les milieux de travail, les jeunes adultes accordent davantage d'importance aux considérations économiques qu'à celles linguistiques. Nous soulignerons également qu'après avoir vécu de la discrimination (racisme, linguicisme, intolérance religieuse), les jeunes adultes des minorités visibles, principalement francophones, tendent à se rediriger vers les milieux anglophones. En conclusion, nous discuterons des implications sociales et politiques de ces résultats.

\section{L'entrée sur le marché du travail et les jeunes adultes issus de l'immigration}

Plusieurs recherches menées sur l'intégration socioéconomique des jeunes adultes issus de l'immigration dans leur pays d'accueil ont souligné des difficultés d'insertion professionnelle vécues au fil de leurs expériences d'orientation (Brinbaum et Guégnard 2012; Brinbaum et Primon 2013). Plus spécifiquement, dans les recherches menées en contextes canadien 
et québécois, cette problématique a été interprétée sous l'angle de la théorie du capital humain, où sont prises en compte les variables liées à la qualification et à l'expérience professionnelle (Adamuti-Trache et al. 2013; Bastien et Bélanger 2010). Le taux de chômage élevé et la surreprésentation des immigrants, principalement des minorités visibles, dans les emplois précaires et sous-qualifiés ont attiré l'attention des chercheurs qui ont soulevé la question de la discrimination systémique à l'endroit, notamment, des personnes issues des minorités visibles (Bourhis 2008). Kamanzi (2012) met l'accent sur le niveau de qualification élevé des membres des minorités visibles par rapport à l'emploi qu'ils occupent, et ce, en dépit des diplômes canadiens qu'ils ont obtenus. La sous-représentation de certains jeunes adultes issus de l'immigration dans quelques filières professionnelles, de même que le sentiment de discrimination et d'injustice qu'ils peuvent ressentir dans le cadre de leurs orientations professionnelles ont également été soulevés dans certaines recherches (Brinbaum et Primon 2013; Potvin et Leclercq 2014).

Au Québec, le mandat de socialisation linguistique en français (basé sur la loi 101) influence le parcours scolaire des jeunes issus de l'immigration du primaire jusqu'à leur insertion sur le marché du travail. Étant donné que leur intégration économique passe par l'éducation, leurs choix au postsecondaire ont une incidence sur leur insertion professionnelle (Dronkers 2010). La négociation entre les élèves, leur famille et les structures scolaires et sociales se répercute sur le processus de construction de leur orientation professionnelle (Dubet et Martuccelli 1996; Dubet 1994). Le marché du travail québécois est la scène d'une concurrence entre deux langues, l'anglais et le français. La situation de Montréal est encore plus saillante, puisque c'est là où la majorité des immigrants s'installent et qu'il s'agit d'une ville marquée par un marché du travail bilingue (Bélanger et al. 2011). Certaines études soulignent que les orientations linguistiques postsecondaires des jeunes Montréalais issus de l'immigration déterminent en grande partie leurs orientations linguistiques professionnelles, selon leur anticipation des normes langagières du marché du travail (Forcier 2011; Pagé et Lamarre 2010). Or, la lecture prospective du milieu du travail peut influencer leurs choix en cette matière (Bélanger et al. 2011; Langlois 2009; Pagé et Lamarre 2010).

Toutefois, les recherches se concentrent généralement sur l'accès à l'emploi pour appréhender l'intégration sociale de ces jeunes. Pourtant, d'autres dimensions jalonnent leur parcours professionnel (intégration linguistique, sociale, rapports ethnoculturels, aspirations, etc.) (Kanouté et al. 2008; Ogbu et Simmons 1998; Potvin et Leclercq 2014). Le sentiment de discrimination et d'injustice que ces jeunes vivent et rapportent dans leur orientation professionnelle est relié au fonctionnement des 
systèmes éducatifs des pays d'accueil (Brinbaum et Primon 2013). Les indicateurs quantitatifs ne permettent pas de comprendre de manière approfondie d'autres dimensions du processus d'orientation des jeunes adultes issus de l'immigration, comme leurs rapports positifs ou conflictuels à l'école et à la société d'accueil (Douglas et Saenz 2008), l'expérience de la discrimination (Lafortune et Kanouté 2007; Potvin et Leclercq 2014) ou les rapports d'exclusion qu'ils vivent dans leurs expériences scolaires et extrascolaires (Breton 2015, 2012). Ainsi, la négociation des rapports ethnoculturels fait partie de leur expérience de vie, dans les aspects tant scolaires que professionnels. Et pourtant, aucune étude à ce jour n'a traité du rôle de ces rapports dans le déroulement des expériences d'orientation professionnelle des jeunes adultes issus de l'immigration au Québec et à Montréal.

Il semble donc crucial d'explorer les expériences d'orientation professionnelle de ces jeunes adultes sous un angle interprétatif qui permet de mieux saisir la complexité des dimensions sociales qui les structurent, et ce, en reconnaissant leur travail de négociation vis-à-vis des déterminations sociales et des relations sociales. Afin d'atteindre cet objectif, nous nous focalisons sur les questions suivantes: quelles sont les dimensions sociales qui interviennent dans les parcours professionnels des jeunes adultes issus de l'immigration? Comment, en négociant ces dimensions, construisent-ils leurs expériences d'orientation professionnelle? Comment la négociation des rapports interculturels s'articule-t-elle par rapport à leur choix d'orientation professionnelle?

\section{Le cadre d'analyse: une conception relationnelle de l'expérience d'orientation}

Nous avons choisi d'adopter une approche relationnelle pour conceptualiser l'expérience d'orientation professionnelle. Dans cette approche, une médiation s'établit entre les contraintes structurelles de la société et la capacité de l'acteur à négocier son autonomie, et ce, en faisant le pont entre les conceptions interactionniste et structuraliste de l'expérience de vie (Liu et Emirbayer 2016). La conception interactionniste permet de penser la construction des expériences humaines comme un processus, en reconnaissant le pouvoir de l'acteur à transformer les structures sociales par ses interprétations collectivement construites. Dans ce sens, les interactions sociales ne se limitent pas aux rapports de lutte sociale, elles peuvent être sujettes aux rapports de coopération et de compétition (Abbott 2005). La conception structuraliste permet, quant à elle, d'analyser les structures de la vie sociale sur lesquelles l'acteur n'a pas de prise. Dans cette approche, les analyses des expériences sont limitées à celle des 
rapports de pouvoir qui structurent la société (Bourdieu 2007). Cette approche permet de considérer davantage les déterminations d'ordre macrosocial (Liu et Emirbayer 2016). L'approche relationnelle, à la croisée des visions interactionniste et structuraliste, permet de saisir les singularités des expériences de vie dans leur complexité et dans leur hétérogénéité. Son objectif est d'analyser la complexité des dimensions, tant relationnelles que contextuelles, dans les relations qu'établit l'acteur avec autrui et avec les normes de la société (ibid.). Ainsi, dans notre conceptualisation relationnelle de l'expérience, les interactions entre les individus, les groupes sociaux et les groupes professionnels sont nécessairement reliées aux rapports de pouvoir qui structurent une société. De plus, l'acteur social, pour construire ses expériences de vie et pour leur donner un sens subjectif, négocie à la fois les rapports de pouvoir, les interactions sociales et son image de soi. Partant de cette conceptualisation de l'expérience de vie, nous cherchons à comprendre le processus de construction et de reconstruction des choix d'orientation professionnelle. Ainsi, deux dimensions conceptuelles ressortent du cadre théorique de la recherche: les «déterminations sociales négociées par l'acteur» et les «relations sociales négociées par l'acteur». Ces dimensions nous orientent vers l'opérationnalisation du cadre conceptuel, ainsi que vers les stratégies d'analyse des résultats.

\section{Les déterminations sociales négociées par l'acteur}

Cette dimension permet de focaliser l'analyse sur le travail de négociation de l'acteur vis-à-vis des déterminations sociales afin de construire ses expériences d'orientation professionnelle. Les expériences humaines ne sont «ni le produit d'une pure intériorité, ni l'effet d'un contexte extérieur à l'être humain, elles sont le fruit des relations d'interdépendance passées et présentes qui sont exercées et continuent à s'exercer sur lui» (Lahire 2013: 41). Ainsi, cet acteur «pluriel» est lui-même «le produit de l'expérience des contextes sociaux multiples et hétérogènes » (Lahire 2005:42). Or, les conditions sociales qui régissent les expériences sociales peuvent être différentes d'un acteur à l'autre. Cette dimension fournit ainsi les dimensions microsociologiques et macrosociologiques à partir desquelles les acteurs sociaux construisent leurs expériences de vie (Lahire 2013) en leur attribuant un sens subjectif (Becker 2008). Il s'agit donc de comprendre comment les jeunes adultes négocient leur marge de manœuvre dans un espace social structuré par des déterminations sociales, autant macrosociologiques que microsociologiques. La prise en compte du travail de négociation des jeunes adultes sur ces deux dimensions nous oriente vers les types variés d'expériences d'orientation qui se construisent 
autour des logiques d'action que les jeunes adultes empruntent (Dubet 1994). Le concept de logique d'action permet de creuser différents types d'expériences humaines (Becker 2008; Pilote 2005), telles que la performance scolaire, la construction identitaire ou, dans le cas de notre étude, l'expérience d'orientation. Ainsi, nous pouvons déceler les logiques d'action, voire les logiques d'orientation variées qu'empruntent les acteurs sociaux par rapport aux conditions et aux relations sociales qui structurent leurs expériences (Lahire 2013).

\section{Les relations sociales négociées par l'acteur}

Cette dimension permet de saisir comment les jeunes adultes, en tant que minoritaires, négocient leurs relations sociales avec les Québécois francophones du groupe majoritaire tout au long de leur processus d'orientation (Lamont et Molnár 2002; Liu et Emirbayer 2016). À travers cette dimension, nous nous penchons sur les représentations que les acteurs se font de leur vécu d'interactions avec les Québécois francophones de naissance. Ce vécu des acteurs nous sert de dimension d'analyse, et non pas d'objet de recherche en soi. Cela permet notamment de prendre en compte le rôle des rapports de pouvoir qu'attribue l'acteur à l'ensemble de ses expériences d'orientation (Blumer 1958).

Pour conclure, les objectifs de recherche guidant notre démarche méthodologique sont les suivants: 1) repérer les dimensions sociales intervenant dans le processus d'orientation professionnelle des jeunes adultes issus de l'immigration à Montréal; 2) décrire le rôle de la dynamique relationnelle entre ces jeunes adultes et les Québécois francophones par rapport à leur processus d'orientation; 3) cerner les logiques multiples d'action que ces jeunes mettent en œuvre pour construire leur expérience d'orientation professionnelle.

C'est donc à partir de ce cadre conceptuel, axé sur les expériences hétérogènes et singulières des acteurs sociaux construites au croisement des relations et des déterminations sociales, que nous analyserons les données qualitatives recueillies auprès des jeunes adultes.

\section{L’approche méthodologique}

Les données présentées dans cet article proviennent d'une étude qualitative basée sur les récits de vie de jeunes adultes issus de l'immigration (Bertaux 2010). Les entretiens ont été réalisés de juillet 2015 à mars 2016. Parmi les vingt-cinq répondants, treize sont arrivés au pays alors qu'ils avaient l'âge de fréquenter le secondaire et les douze autres ont fait leur primaire au Québec. Parmi ces derniers, deux sont nés à Montréal et les 
dix autres sont arrivés au pays vers l'âge de sept ans. Les répondants comptaient au moins deux ans d'expérience sur le marché du travail ${ }^{1}$. Nous avons interviewé dix-huit femmes et sept hommes, tous âgés de 24 à 35 ans. Parmi les répondants, deux étaient originaires d'Afrique centrale, un d'Afrique de l'Ouest, deux d'Afrique du Nord, six d'Amérique du Sud, trois d'Asie de l'Est, deux d'Asie du Sud-Est, un d'Europe, deux d'Europe de l'Est et six du Moyen-Orient. Parmi les vingt-cinq jeunes adultes de ce corpus, vingt et un appartiennent aux différentes catégories de minorités visibles. Il est à noter que nous nous basons sur la définition du gouvernement du Canada pour définir la catégorie « minorités visibles ${ }^{2} »$. Ainsi, parmi les vingt et une personnes du corpus qui appartiennent à une minorité visible, deux sont dans la catégorie "Arabe» (Algérie, Tunisie); trois dans la catégorie «Asiatique du Sud-Est» (Cambodge, Vietnam); six dans la catégorie «Asiatique occidentale»(Iran, Syrie); deux dans la catégorie «Latino-Américain» (Uruguay, Pérou); un dans la catégorie «Chinois» et sept dans la catégorie «Noir» (Haïti, Congo, Cameroun, Guinée). Par ailleurs, les jeunes adultes du corpus qui n'appartiennent pas à la catégorie "minorités visibles» sont originaires de la Russie (1), de la Moldavie (1), de la Roumanie (1) et de l'Italie (1).

En ce qui a trait au parcours scolaire des vingt-cinq répondants du corpus, seize se sont orientés vers un cégep francophone et six vers un cégep anglophone; trois répondants se sont orientés vers un cégep anglophone après avoir passé la moitié de leur programme dans un cégep francophone. En ce qui concerne le choix du type de programme au collégial (préuniversitaire ou technique), vingt-deux des vingt-cinq jeunes ont choisi un programme préuniversitaire, deux ont opté pour une technique et un a fait à la fois un programme préuniversitaire et une technique.

Pour ce qui est du parcours postsecondaire au niveau universitaire, vingt-deux répondants du corpus ont poursuivi leurs études jusqu'à cette étape. Parmi eux, douze répondants ont obtenu un diplôme de baccalauréat, trois ont obtenu un diplôme de maîtrise, cinq répondants terminaient leur baccalauréat au moment de l'entrevue et deux répondants ont abandonné leurs études universitaires vers la fin de leur programme. Seuls deux répondants ayant choisi une technique ${ }^{3}$ au postsecondaire n'étaient pas entrés à l'université. Au moment de l'entrevue, ils avaient commencé à travailler dans un domaine lié à leur diplôme collégial.

En ce qui concerne le parcours professionnel des répondants du corpus, dix d'entre eux travaillent dans un milieu anglophone, sept sont dans un milieu de travail francophone et huit dans un milieu bilingue. Les types d'emplois des répondants du corpus sont les suivants : agent de placement, agent au gouvernement, adjoint à la direction, agent de vente, 
comptable, consultant, chercheur, esthéticienne, dessinateur, enseignant, employé administratif, enquêteur, infirmier, ingénieur, massothérapeute, responsable des finances, secrétaire, aide aux ventes, technicien et tuteur.

Les entretiens de type récits de vie ont généralement duré plus de deux heures chacun. Lors des récits de vie rétrospectifs, les participants ont été invités à narrer leurs expériences de vie à partir du parcours migratoire de leurs parents jusqu'à leur expérience sur le marché du travail montréalais. Les jeunes ont eu le choix de répondre aux questions en français ou en anglais. Les entrevues ont été enregistrées puis retranscrites intégralement.

Après l'analyse des récits de vie, un codage a été effectué à l'aide du logiciel QDA Miner afin de repérer dans les témoignages des répondants les dimensions sociales qui interviennent dans leur processus d'orientation professionnelle. Les dimensions suivantes à l'échelle micro- et macrosociale sont ressorties de cette analyse: «langue maternelle», «vécu de la fratrie», «rapport des parents à la société d'accueil», «aspirations des parents et des jeunes», "vécus de la discrimination » et "dimensions liées au marché du travail ». Par la suite, à l'aide d'une deuxième codification, nous avons analysé le rôle des représentations qu'ils se font de leurs relations avec le Québec et avec les Québécois francophones quant à leur processus d'orientation. Les dimensions suivantes sont ressorties de cette analyse: "perception positive des Québécois francophones», «perception négative des Québécois francophones», «rapport harmonieux avec le Québec » et «rapport conflictuel avec le Québec». Enfin, par une analyse typologique, nous avons croisé les dimensions d'analyse (dynamique relationnelle établie avec les Québécois francophones; dimensions sociales intervenant dans les logiques d'orientation) repérées dans le discours des jeunes adultes.

Lanalyse typologique (Schnapper 1999) fut un outil fécond pour la mise en rapport des témoignages des répondants du corpus sur leurs expériences d'orientation professionnelle. Cela nous permet de construire des idéaux types qui mettent en lumière les conditions sociales semblables sous lesquelles certains types d'expériences humaines se produisent (Becker 1940; Schnapper 1999). Cette approche permet d'interpréter théoriquement les témoignages collectés par les récits et de leur donner un sens conceptuel. Or, la réalité racontée par les narrateurs ne possède pas de réalité théorique, car les individus ne vivent pas leurs expériences de vie à travers une perspective théorique (Atkinson 2007). Il revient donc au chercheur de déceler cette perspective (Becker 1940) que pourraient engendrer ces témoignages.

L'analyse typologique nous a ainsi permis de construire trois types de logiques d'orientation professionnelle. Plus spécifiquement, par «logique», 
nous entendons le sens que donne l'acteur social à ses expériences de vie en négociant le rôle déterminant des dimensions sociales (Lahire 2005) et en attribuant des significations aux interactions qu'il a établies avec les autres acteurs lors du processus de réalisation (Becker 1985). Notre recherche, menée à Montréal, situe les interactions sociales au sein de rapports ethnoculturels bien spécifiques - les rapports entre les jeunes adultes issus de l'immigration et les Québécois francophones (Jenkins 1994; Juteau 2015). De ce fait, la prise en compte du rôle des rapports ethnoculturels dans la construction de l'expérience d'orientation professionnelle permet de contextualiser les logiques d'orientation. La prochaine section vise à expliciter cette typologie.

\section{Une typologie des logiques d'orientation professionnelle}

Les analyses ont mis en évidence trois logiques d'orientation : la «logique d'accès à l'autonomie économique», la «logique d'éloignement du milieu francophone» et la «logique de mobilité géographique anticipée».

\section{La logique d'accès à l'autonomie}

Dans la logique d'accès à l'autonomie, l'acteur social construit son expérience d'orientation professionnelle en choisissant un milieu de travail qui favorise son insertion professionnelle dans un bref délai, et ce, peu importe que la langue de ce milieu soit l'anglais ou le français. Une bonne proportion des répondants du corpus (11) s'inscrivent dans cette logique d'orientation, qui cohabite avec les autres logiques d'orientation professionnelle que nous présenterons dans les prochaines sections. Bien que la logique d'accès à l'autonomie économique constitue la logique principale de onze cas du corpus, quatorze autres cas s'inscrivent seulement en partie dans celle-ci. Les jeunes adultes s'y inscrivant entretiennent des relations aussi bien positives que négatives avec le groupe majoritaire francophone québécois. Ce qui importe dans cette logique, c'est l'accès à l'autonomie économique ou l'accès à la profession. Dans les récits des jeunes s'inscrivant dans cette logique, on entend souvent des propos comme «je voulais travailler pour payer mes affaires, en français ou en anglais, je n'y pensais pas» (Sophie, originaire de Taïwan) ou «j'étais tanné d'être dépendant, je voulais travailler, je voulais gagner ma vie, je voulais fonder une famille» (Frank, originaire de la Chine). Il semble que les anticipations économiques et le désir d'autonomie jouent un rôle central dans la construction de cette logique d'orientation professionnelle. De plus, dans cette logique, avoir un emploi semble jouer un rôle plus capital que des motifs qui seraient liés à la structure linguistique du marché du travail montréalais 
(articulé autour des langues française et anglaise). Les jeunes s'inscrivant dans ce type de logique ont confiance en leurs compétences linguistiques (en français et/ou en anglais) lors de leurs orientations professionnelles. Ils se focalisent davantage sur le domaine de l'emploi offert que sur la langue utilisée en milieu de travail. La logique d'accès à l'autonomie économique se décline selon deux types de visées: "professionnelles» et «économiques», approfondies dans les prochaines sections.

Les visées professionnelles: agencer sa formation avec le domaine de l'emploi

Les visées professionnelles correspondent au désir de trouver un emploi dans son domaine de formation universitaire. Parmi les onze jeunes adultes du corpus qui ont construit leur expérience d'orientation professionnelle dans la logique d'accès à l'autonomie économique, sept démontrent des visées professionnelles. Ces jeunes adultes, comme l'explique Sitaa, originaire d'Haïti, «tentent de profiter de leur formation postsecondaire et de la valoriser » pour trouver un emploi dans leur domaine de formation. De ce fait, comme le raconte Frank (originaire de la Chine), «l'acquisition d'une expérience professionnelle dans leur domaine de formation» est au cœur de leurs visées.

Ces jeunes adultes tentent d'agencer leur formation postsecondaire avec leur domaine d'emploi. Soulignons à nouveau que la langue du milieu du travail ne paraît pas peser dans ces visées professionnelles. Il semble que les aspirations de ces jeunes adultes (et non pas celles que leurs parents entretiennent pour eux) interviennent davantage dans le développement de leurs visées professionnelles. L'analyse des récits que font ces jeunes adultes (7) permet de constater qu'ils ont vécu une insertion professionnelle relativement facile. Autrement dit, les entrevues de recrutement, l'attitude des employeurs, les démarches administratives semblent avoir facilité, selon eux, leur insertion.

Les visées économiques: ne pas réussir à agencer sa formation avec le domaine de l'emploi

Les visées économiques se traduisent par la tendance de l'acteur à se trouver un emploi le plus rapidement possible sans essayer de mettre à profit son domaine de formation ou sans réussir à le faire. Parmi les onze jeunes adultes du corpus qui ont construit leur expérience d'orientation professionnelle par la logique d'accès à l'autonomie économique, quatre cas démontrent plus spécifiquement des visées économiques. Ces jeunes trouvent que le marché du travail montréalais est un marché difficile pour les jeunes issus de l'immigration.

Prenons l'exemple de Jimmy, originaire d'Italie, et de Fabrice, originaire d'Haïti, qui ont abandonné leurs études universitaires vers la fin de 
leurs programmes. Après leur abandon, ces deux répondants ont trouvé des emplois en s'appuyant sur leurs diplômes collégiaux, mais dans des domaines différents qui ne sont pas associés à leur formation collégiale. Ils ont souvent changé d'emplois et travaillé dans des domaines ou milieux aux usages linguistiques variés. Comme l'explique Jimmy, «on dirait que j'essayais justement de survivre parce que je n'avais pas d'intérêt particulier $»$. Jimmy et Fabrice insistent sur la présence de discrimination dans le marché du travail montréalais, sur le fait qu'il est difficile de se trouver un emploi dans un contexte de compétition avec les Québécois francophones. De fait, selon ces jeunes adultes, certaines dimensions du marché du travail montréalais sont contraignantes pour leur processus d'insertion professionnelle. En outre, ils ont l'impression de posséder moins de marge de manœuvre pour trouver un emploi lié à leur formation postsecondaire.

\section{La logique d'éloignement du milieu francophone}

Dans cette logique, l'acteur social construit son expérience d'orientation professionnelle en choisissant de s'éloigner, de manière délibérée, des milieux francophones. Dans notre corpus, dix cas s'inscrivent dans cette logique. Parmi ceux-ci, six ont le français comme langue maternelle, deux le russe, un l'arabe et un le persan. Ces répondants entretiennent une relation négative avec le groupe majoritaire: l'expérience d'une forme de discrimination (linguicisme, racisme, intolérance religieuse) est constitutive des récits qu'ils font de leurs interactions avec cet autre groupe. La colère et la tristesse sont les émotions qui teintent les représentations qu'ils se font de leurs interactions avec le groupe majoritaire. Parmi les francophones, ce type d'orientation illustre une bifurcation flagrante par rapport aux logiques précédentes qui ont ponctué leur parcours. Concrètement, malgré le fait que «travailler en français soit moins contraignant pour eux» (car ils maîtrisent mieux la langue française), ils préfèrent «s'éloigner le plus possible des Québécois francophones» (Stim, originaire du Cameroun). Tiam, originaire de la Guinée, a poursuivi toutes ses études postsecondaires dans le secteur francophone. Toutefois, il s'oriente vers un milieu de travail anglophone. Les représentations de Tiam mettent en exergue le vécu de la discrimination raciale. Tiam est «déterminé à ne pas travailler dans un milieu francophone » (Sophie, originaire de Taïwan):

Moi, je me faisais dire par des patients, je ne veux pas que ce soit un Noir qui me touche. J'avais travaillé à l'hôpital Saint-Luc comme préposé, et j'ai une formation de six semaines. Le gars qui me formait, il s'assoyait au poste, il ne faisait rien, il me donnait les cas les plus lourds et il disait au patient d'écrire si je fais un bon travail ou pas. Donc, je me sentais surveillé, je me 
sentais traqué [...] quand tu es Noir, quand tu es immigrant, et que tu parles, ça fait doublement peur [...]. J'avais eu un ami qui m'a dit il faut que tu ailles à l'hôpital juif, tu vas être bien à l'hôpital juif. Dans un hôpital anglophone, c'est plus libéral, les gens sont plus ouverts, il y a beaucoup d'immigrants, et ça ne fonctionne pas pareil. Je suis allé dans les milieux anglophones et, depuis, je travaille là. Le racisme existe aussi dans le milieu anglophone, je veux dire ça n'enlève pas, mais le rapport au racisme il est différent, il est plus violent dans le milieu francophone que dans le milieu anglophone. Il est plus direct dans le milieu francophone que dans le milieu anglophone [...] (Tiam, originaire de la Guinée).

Le vécu de l'intolérance religieuse façonne l'orientation professionnelle de Mouna, originaire de la Tunisie. Pendant son entrevue, au moment où elle se projette dans le futur, elle insiste sur le fait qu'elle ne va «jamais travailler dans un milieu francophone, jamais, c'est fini avec les Québécois ». Comme Tiam, Mouna se positionne contre certaines normes de la société québécoise, contre le projet de Charte des valeurs québécoises et l'idée de l'indépendantisme québécois. Stim, originaire du Cameroun, s'oriente également vers un milieu de travail anglophone après avoir fait toutes ses études postsecondaires en français. L'expérience de la discrimination raciale est constitutive de son parcours de vie et de celui de son père. Stim trouve qu'en comparaison avec les francophones du Québec, les anglophones intègrent plus les immigrants:

Je suis plus à l'aise en français. C'est ma langue, genre je ne sais pas, je trouve ça plus beau, ça fait plus moi, tu vois. Mais les anglophones, sérieusement, ça, c'est un truc que j'ai remarqué... Les anglophones, ils sont moins portés sur les préjugés. Même s'ils ont des préjugés, leurs préjugés sont au niveau personnel, au niveau professionnel ils restent professionnels, donc ça veut dire que si tu es Noir ou Blanc ou Asiatique, peu importe, si tu as les qualifications, ils ne choisissent pas que la personne "ah, lui, il me ressemble plus», eux ils choisissent, "ah, lui, il est plus qualifié». Ils vont t'embaucher et c'est comme ça. Ça, c'est un truc que j'adore chez les anglophones [...] (Stim, originaire du Cameroun).

Ainsi, la logique d'éloignement permet d'observer notamment la tendance de certains répondants du corpus (faisant principalement partie de la catégorie «minorités visibles ») à s'insérer dans les milieux de travail anglophones en raison de la discrimination vécue dans les milieux francophones.

\section{La logique de mobilité géographique anticipée}

Dans la logique de mobilité géographique anticipée, l'acteur social construit son expérience d'orientation professionnelle selon l'anticipation 
d'une mobilité géographique vers les autres provinces du Canada ou vers d'autres pays. Parmi les jeunes adultes du corpus, quatre s'inscrivent dans cette logique. Les représentations que ces jeunes adultes se font de leurs interactions avec le groupe majoritaire permettent d'observer leur perception négative du Québec et des Québécois francophones. Prenons l'exemple de Sophie, originaire de Taïwan, qui a terminé ses études dans une université anglophone. Par la suite, elle a trouvé un emploi dans un milieu anglophone. L'objectif de «quitter un jour le Québec et d'aller vivre ailleurs» (Sophie, originaire de Taïwan) constitue son motif principal pour travailler dans un milieu anglophone. Cette répondante souligne «que je visais un pays anglophone». Ainsi, vers la fin de ses études universitaires, elle a planifié son futur de sorte que son expérience professionnelle dans les milieux de travail anglophones facilite son projet de mobilité. Précisons que la logique de la mobilité géographique anticipée prend une grande importance dans son récit; toutefois, nous avons observé qu'elle s'inscrivait également, dans une moindre mesure, dans la logique d'éloignement du milieu francophone.

Trois répondantes qui ont d'abord eu une expérience de travail dans des milieux francophones se situent également dans cette logique. Elles ont abandonné leurs emplois en milieu francophone pour en trouver un en milieu anglophone, puisqu'elles envisagent «de vivre et de travailler hors du Québec» (Lucia, originaire d'Uruguay). Il semble que ces jeunes adultes n'ont pas réussi, malgré leurs efforts, à se sentir incluses dans le groupe majoritaire.

En somme, la construction des idéaux types des logiques d'orientation professionnelle nous a permis de dégager trois logiques d'orientation professionnelle (logique d'accès à l'autonomie économique, logique d'éloignement du milieu francophone, et logique de mobilité géographique anticipée). Cette typologie permet d'observer les particularités de cette expérience par rapport à l'hétérogénéité des dimensions sociales qui interviennent dans les expériences humaines. Ainsi, chaque individu négocie de façon singulière les dimensions qui interviennent dans son expérience d'orientation. Et ce travail de négociation se réalise au cœur des interactions qui régissent la négociation des rapports ethnoculturels avec le groupe majoritaire francophone. Dans les lignes qui suivent, nous tentons d'interpréter la portée sociale et scientifique de ces idéaux types.

\section{Discussion et conclusion}

Ces trois logiques d'orientation professionnelle ouvrent la réflexion sur la complexité et l'hétérogénéité de l'expérience d'orientation professionnelle des jeunes adultes, une complexité qui ne se saisit qu'en la situant au 
croisement de plusieurs éléments sociaux, comme la dynamique relationnelle et le sentiment de vivre des discriminations. La logique d'éloignement du milieu francophone illustre notamment cette complexité. Elle est empruntée par les jeunes adultes qui, se sentant exclus du groupe des Québécois francophones, s'éloignent des milieux de travail francophones pour s'orienter vers les milieux anglophones. Paradoxalement, cette logique est empruntée principalement par les jeunes adultes de minorités visibles noires (Haïtiens, Guinéens et Camerounais) et arabes (Tunisiens et Algériens) qui ont le français comme langue maternelle et qui ont de la difficulté à travailler en langue anglaise. Soulignons que la catégorie Arabe comprend deux femmes voilées du corpus, une Tunisienne et une Algérienne. Ces constats attirent l'attention sur le poids du sentiment d'exclusion et de discrimination dans la construction de la logique d'éloignement du milieu francophone, et non pas sur le poids des caractéristiques individuelles et familiales (Guìrra 2016; Mc Andrew et al. 2011). Autrement dit, ce n'est pas sous l'effet de la langue maternelle ou des aspirations des jeunes et de leurs parents que les jeunes adultes s'éloignent des milieux francophones. C'est principalement leur rapport conflictuel vis-à-vis du groupe majoritaire et leurs vécus de discrimination qui semblent expliquer ce désir d'éloignement. Les trois types de logiques d'orientation professionnelle attirent l'attention sur le rôle de la dynamique relationnelle, établie avec les Québécois francophones lors du processus d'orientation: il s'agit d'une dimension sociale qui a peu été prise en compte jusqu'à présent dans les études quantitatives et qualitatives portant sur l'accès au marché du travail chez les jeunes adultes issus de l'immigration (Brinbaum et Primon 2012; Kamanzi 2012; Mc Andrew et al. 2011). La tendance des jeunes adultes de minorités visibles des catégories Noir et Arabe à développer des logiques d'éloignement du milieu francophone et de mobilité géographique anticipée invite à réfléchir aux interactions entre les logiques propres aux individus (par exemple, le désir d'éloignement) et les normes relatives au marché du travail (Trottier 2005). Il s'avère que les jeunes adultes, en empruntant ces logiques, tentent de se libérer, autant qu'ils le peuvent, des contraintes ressenties et liées au marché du travail montréalais (discrimination ressentie). Autrement dit, les réorientations vers les milieux professionnels anglophones traduisent le rôle crucial du vécu du racisme, du linguicisme et de l'intolérance religieuse dans la construction de leurs logiques d'orientation professionnelle.

Les résultats de notre recherche invitent à nuancer les résultats des études statistiques qui soulignent que la réalité bilingue du marché du travail montréalais pourrait favoriser les orientations vers les milieux de travail anglophones (Bélanger et al. 2011). En effet, les représentations que 
les jeunes adultes issus de l'immigration se font de la réalité du marché du travail montréalais dressent le portrait d'un marché structuré par des milieux de travail jugés plus inclusifs (les milieux anglophones) ou moins inclusifs (les milieux francophones). Ces résultats, replacés dans les débats autour de l'influence du choix de la langue anglaise au postsecondaire sur les orientations professionnelles subséquentes vers les milieux de travail anglophones (Forcier 2011), laissent à penser que la tendance à travailler dans les milieux anglophones pourrait être le résultat d'un sentiment d'exclusion vécu dans les milieux francophones, du moins pour les jeunes de minorités visibles. Les résultats présentés invitent ainsi à nuancer les constats des études affirmant que les jeunes choisissent comme langue du travail celle dans laquelle ils ont été scolarisés au postsecondaire (Forcier 2011; Pagé et Lamarre 2010).

L'ensemble de ces logiques d'orientation professionnelle souligne également l'importance de l'accès à l'autonomie économique chez les jeunes adultes issus de l'immigration, les considérations liées aux choix linguistiques des milieux du travail devenant moins saillantes pour certains. Cette tendance est présente dans les trois logiques (d'accès à l'autonomie économique, d'éloignement du milieu francophone et de mobilité géographique anticipée). Il nous semble que l'importance des types reliés à la nécessité d'accès à l'autonomie économique pourrait s'expliquer par l'importance sociétale accordée à l'insertion dans le marché du travail après la sortie du système éducatif (Dubar 2001). Toutefois, les résultats démontrent que les jeunes adultes cherchent vivement à transformer leurs visées économiques en visées professionnelles au fil de leur parcours professionnel, une réalité qui s'illustre dans leurs réorientations et leurs bifurcations (changement de domaine ou de milieu travail). Les résultats attirent ainsi l'attention sur le rôle important des normes perçues ou de l'expérience de discrimination vécue sur le marché du travail quant aux logiques d'orientation professionnelle (Chicha 2012). Ajoutons qu'ils permettent de développer un regard complémentaire et plus affiné sur les données statistiques sur l'égalité d'accès à l'emploi auprès de la population immigrante au Québec (Secrétariat du Conseil du trésor du Québec [SCTQ] 2015). Les résultats de notre étude sur vingt-cinq jeunes adultes issus de l'immigration montrent que seulement une minorité d'entre eux (7) estiment avoir développé des visées professionnelles où leur domaine d'emploi correspond à leur domaine de formation.

Les discriminations vécues par les jeunes adultes issus de l'immigration de différents groupes minoritaires, dans différentes sphères de leur vie scolaire et sociale, font réfléchir au potentiel de la société québécoise à reproduire des rapports d'exclusion dans ses institutions éducatives et 
productives. Cette dynamique semble nuire au vivre-ensemble souhaité dans les politiques éducatives. Ainsi, dans des recherches quantitatives futures, il serait pertinent de porter une attention particulière aux vécus de discrimination et au sentiment d'exclusion que ressentent ces individus dans leurs expériences professionnelles au sein de la société québécoise. Il serait également pertinent de mener des études comparatives sur ce sujet entre le Québec et d'autres provinces anglophones du Canada.

\section{Notes}

1. Nous avons insisté sur une expérience de deux ans sur le marché du travail, pour qu'ils puissent avoir un certain recul réflexif sur cette expérience et sur les choix postsecondaires et professionnels qu'ils ont effectués.

2. Selon Statistique Canada (2011), «on entend par minorités visibles, le groupe de minorités visibles auquel le répondant appartient. Selon la Loi sur l'équité en matière d'emploi, font partie des minorités visibles "les personnes, autres que les Autochtones, qui ne sont pas de race blanche ou qui n'ont pas la peau blanche". La variable minorités visibles comprend les catégories suivantes: Sud-Asiatique, Chinoise, Noire, Philippine, Latino-Américaine, Arabe, Asiatique du Sud-Est, Asiatique occidentale, Coréenne, Japonaise, Minorité visible, n.i.a. (signifiant "non incluse ailleurs"), Minorités visibles multiples et pas une Minorité visible» (p. 4).

3. Ces répondants pensent retourner aux études.

\section{Bibliographie}

Abbott, A., 2005. «Ecologies and Fields», University of Chicago. http://home.uchicago. edu/aabbott/Papers/BOURD.pdf [consulté le 10 mai 2016].

Adamuti-Trache, M., P. Anisef et R. Sweet, 2013. «Impact of Canadian Postsecondary Education on Occupational Prestige of Highly Educated Immigrants ", Canadian Review of Sociology/Revue canadienne de sociologie, vol. 50, n 2, p. 178-202.

Atkinson, R., 2007. «The Life Story Interview as a Bridge in Narrative Inquiry», Handbook of Narrative Inquiry: Mapping a Methodology, p. 224-245.

Bastien, Y. et A. Bélanger, 2010. Un portrait comparatif de la situation de l'emploi chez les immigrants et les minorités visibles dans les RMR de Montréal, OttawaGatineau, Toronto et Vancouver, Montréal. Emploi Québec. http://emploi-metropole.org/wp-content/uploads/2015/03/clienteles_imm_rapport2.pdf [consulté le décembre 2012].

Becker, H., 1940. «Constructive Typology in the Social Sciences», American Sociological Review, vol. 5, n 1, p. 40-55.

Becker, H. S., 2008. Outsiders. New York, Simon \& Schuster.

Becker, H. S., 1985. Outsiders: études de sociologie de la déviance. Paris, Éditions Métailié.

Bélanger, A., P. Sabourin et R. Lachapelle, 2011. «Une analyse des déterminants de la mobilité linguistique intergénérationnelle des immigrants allophones au Québec », Cahiers québécois de démographie, vol. 40, n 1, p. 113-138.

Bertaux, D., 2010. Le récit de vie. Paris, Armand Colin. 
Blumer, H., 1958. « Race Prejudice as a Sense of Group Position», Pacific Sociological Review, vol. 1, n 1, p. 3-7.

Bourdieu, P., 2007. La distinction: critique sociale du jugement. Paris, Éditions de Minuit.

Bourhis, R., 2008. The Vitality of the English-Speaking Communities of Quebec: From Community Decline to Revival. Centre d'études ethniques des universités montréalaises (CEETUM) et Canadian Institute for Research on Linguistic Minorities (CIRLM). www.icrml.ca/images/stories/documents/en/Richard_Y_Bourhis/chapitre_9_bourhis_landry.pdf [consulté le 2 juin 2014].

Breton, C., 2015. «Making National Identity Salient: Impact on Attitudes Toward Immigration and Multiculturalism», Canadian Journal of Political Science/Revue canadienne de science politique, vol 48, $\mathrm{n}^{\circ} 2$, p. 357-381.

Brinbaum, Y. et J. L. Primon, 2013. «Parcours scolaires et sentiment d'injustice et de discrimination chez les descendants d'immigrés», Économie et statistiques, $\mathrm{n}^{\circ} 464$ 465-466, p. 215-243.

Brinbaum, Y. et C. Guégnard, 2012. «Parcours de formation et d'insertion des jeunes issus de l'immigration au prisme de l'orientation ", Formation emploi. Revue française de sciences sociales, vol. 118, p. 61-82.

Chicha, M. T., 2012. «Discrimination systémique et intersectionnalité: la déqualification des immigrantes à Montréal», Canadian Journal of Women and the Law, vol. $24, n^{\circ} 1$, p. 82-113.

Comité de gestion de la taxe scolaire de l'île de Montréal (CGTSIM), 2016. Portrait socioculturel des élèves inscrits dans les écoles publiques de l'île de Montréal. http:// cgtsim.qc.ca/fr/documents-site-web/311-portrait-socioculturel-2017-2018/file [consulté le 4 avril 2017].

Douglas, K. M. et R. Saenz, 2008. «No Phone, No Vehicle, No English, and No Citizenship: The Vulnerability of Mexican Immigrants in the United States », in A. J. Hattery, D. G. Embrick et E. Smith (dir.), Globalization and America: Race, Human Rights, and Inequality. New York, SAGE Publications, p. 161-180.

Dronkers, J., 2010. Positive but also Negative Effects of Ethnic Diversity in Schools on Educational Achievement? An Empirical Test with Cross-National PISA Data. Communication présentée à la conférence Integration and Inequality in Educational Institutions, 24 et 25 septembre, Brême.

Dubar, C., 2001. «La construction sociale de l'insertion professionnelle», Éducation et Sociétés, vol. 1, n 7, p. 23-36.

Dubet, F., 1994. Sociologie de l'expérience. Paris, Le Seuil.

Dubet, F. et D. Martuccelli, 1996. À l'école. Sociologie de l'expérience scolaire. Paris, Le Seuil.

Forcier, H., 2011. Recherche sur la fréquentation linguistique des cégeps. Montréal, Centrale des syndicats du Québec (CSQ). http://www.lacsq.org/fileadmin/user upload/csq/documents/documentation/notes_rapports_de_recherche/recherche_ frequentation_linguistique_cegeps.pdf [consulté le 10 octobre 2013].

Gurría, A., 2016. PISA Results in Focus. OECD 2015. https://www.oecd.org/pisa/pisa2015-results-in-focus.pdf [consulté le 7 avril 2017].

Jenkins, R., 1994. « Rethinking Ethnicity: Identity, Categorization, and Power », Ethnic and Racial Studies, vol. 17, n² 2, p. 197-223. 
Juteau, D., 2015. L'ethnicité et ses frontières (2e éd.). Montréal, Les Presses de l'Université de Montréal.

Kamanzi, P. C., 2012. «L'emprise des titres scolaires sur l'emploi chez les membres de minorités visibles: comparaison entre le Québec et l'Ontario», Recherches sociographiques, vol. 53, n 2 2, p. 315-336.

Kamanzi, P. C. et J. Murdoch, 2011. «L'accès à un diplôme universitaire chez les immigrants", in G. Lafortune et F. Kanouté (dir.), Familles québécoises d'origine immigrante. Les dynamiques de l'établissement. Montréal, Les Presses de l'Université de Montréal, p. 145-158.

Kanouté, F. et al., 2008. «Familles et réussite scolaire d'élèves immigrants du secondaire", Revue des sciences de l'éducation, vol. 34, n² 2, p. 265-289.

Lafortune, G. et F. Kanouté, 2007. «Vécu identitaire d'élèves de $1^{1 \text { èe }}$ et de $2^{\mathrm{e}}$ génération d'origine haïtienne», Revue de l'Université de Moncton, vol. 38, n² 2, p. 33-71.

Lahire, B., 2013. Dans les plis singuliers du social. Individus, institutions, socialisations. Paris, La Découverte.

Lahire, B., 2005. L'homme pluriel. Les ressorts de l'action. Paris, Armand Colin.

Lamont, M. et V. Molnár, 2002. "The Study of Boundaries in the Social Sciences", Annual Review of Sociology, vol. 28, p. 167-195.

Langlois, S., 2009. «La place du français au Québec: bilan nuancé», in M. Fahmy (dir.), L'état du Québec 2009. Tout ce qu'il faut savoir sur le Québec d'aujourd'hui. Montréal, Fides, p. 105-112.

Liu, S. et M. Emirbayer, 2016. «Field and Ecology», Sociological Theory, vol. 34, n 1, p. 62-79.

Mc Andrew, M., 2010. Les majorités fragiles et l'éducation. Montréal, Presses de l'Université de Montréal.

Mc Andrew, M. et al., 2011. «Les carrières scolaires des jeunes allophones à Montréal, Toronto et Vancouver: une analyse comparative», Journal of International Migration and Integration, vol. 12, $\mathrm{n}^{\circ}$ 4, p. 495-515.

Ministère de l'Immigration, de la Diversité et de l'Inclusion (MIDI), 2016. Fichesynthèse sur l'immigration au Québec-2015. Québec, gouvernement du Québec. http://www.midi. gouv.qc.ca/publications/fr/recherches-statistiques/FICHE_syn_ an2015.pdf [consulté le 16 février 2017].

Ogbu, J. U. et H. D. Simons, 1998. «Voluntary and Involuntary Minorities : A CulturalEcological Theory of School Performance with Some Implications for Education», Anthropology \& Education Quarterly, vol. 29, n² 2, p. 155-188.

Pagé, M. et P. Lamarre, 2010. L’intégration linguistique des immigrants au Québec. Montréal, IRPP.

Pilote, A., 2005. La construction de l'identité politique des jeunes en milieu francophone minoritaire. Le cas des élèves du centre scolaire communautaire Sainte-Anne à Fredericton au Nouveau-Brunswick. Thèse de doctorat en administration et politiques scolaires, Faculté des sciences de l'éducation, Université Laval.

Potvin, M. et J.-B. Leclercq, 2014. «Facteurs affectant la trajectoire scolaire des jeunes de 16-24 ans issus de l'immigration en formation générale des adultes ", Revue des sciences de l'éducation, vol. 40, n 2, p. 309-349.

Schnapper, D., 1999. La compréhension sociologique. Démarche de l'analyse typologique. Paris, Presses universitaires de France. 
Secrétariat du Conseil du trésor du Québec (SCTQ), 2015. Archives des statistiques en matière d'accès à l'égalité en emploi, 2014-2015. Québec, gouvernement du Québec. https://www.tresor.gouv.qc.ca/fileadmin/PDF/effectif_fonction_publique/groupes_ cibles2014_2015.pdf [consulté le 10 juin 2017].

Statistique Canada, 2011. Guide de référence sur les minorités visibles et le groupe de population, 2011. Ottawa, gouvernement du Canada. http://www12.statcan.gc.ca/ nhs-enm/2011/ref/guides/ 99-010-x/99-010-x2011009-fra.pdf [consulté le 10 octobre 2014].

Statistique Canada, 2016. Classification de la minorité visible. Ottawa, gouvernement du Canada. http://www23. statcan.gc.ca/imdb/p3VD_f.pl?Function=getVD\&TVD= $62052 \& C V D=62053 \& C P V=1 \& C S T=01012004 \& C L V=1 \& M L V=2$ [consulté le 12 avril 2017].

Statistique Canada, 2016. Dictionnaire, Recensement de la population, 2016: Langue maternelle. Ottawa, gouvernement du Canada. http://www12.statcan.gc.ca/censusrecensement/2016/ref/dict/pop095-fra.cfm [consulté le 2 juin 2017].

Trottier, C., 2005. «L'analyse des relations entre le système éducatif et le monde du travail en sociologie de l'éducation: vers une recomposition du champ d'études? », Éducation et Sociétés, vol. 2, n 16, p. 77-97. 